\title{
Errores de refracción y alteraciones acomodativas en niños con dificultades en el aprendizaje
}

\section{Refractive errors and accommodative insufficiency in children with learning difficulties}

\author{
Andrea Rangel-Padilla*, Juan H. Paéz-Garza, Antonella Royero-Alemán, Sylvia De la Rosa-Pacheco y \\ María T. Rodríguez-Neira \\ Escuela de Medicina y Ciencias de la Salud, Tecnológico de Monterrey, Monterrey, Nuevo León, México
}

\begin{abstract}
Resumen
Introducción: Las dificultades en el aprendizaje se consideran la primera causa de fracaso escolar. Se requiere un abordaje multidisciplinario para su diagnóstico y tratamiento. Dentro de la batería de estudios se encuentra la valoración oftalmológica con el objetivo de descartar algún problema asociado que pueda interferir en el proceso de aprendizaje. Existe información limitada sobre el estado visual de los pacientes con dificultades de aprendizaje en nuestra población, por lo que es de importancia evaluar las alteraciones oftalmológicas que con más frecuencia se encuentran. Objetivo: Describir las alteraciones oftalmológicas encontradas en niños con dificultades de aprendizaje referidos para evaluación oftalmológica. Método: Estudio transversal observacional en el que se incluyeron 69 pacientes de 5 a 16 años, referidos desde una institución de educación que brinda apoyo a niños con dificultades de aprendizaje para valoración oftalmológica, en el período comprendido de enero de 2018 a enero de 2019. De los expedientes clínicos se tomaron las siguientes variables: sexo, edad, agudeza visual, alineación ocular, fusión sensorial, estereoagudeza, punto próximo de convergencia, visión de colores, amplitudes acomodativas, refracción ciclopléjica, biomicroscopía, fundoscopía y diagnóstico. Resultados: Se analizaron datos de 69 pacientes con un promedio de edad de 9.3 años ( \pm 3.5 ), de los que 51 eran de sexo masculino. Presentaron alguna alteración oftalmológica 38 pacientes, encontrando errores de refracción en 22, insuficiencia acomodativa en 10 e insuficiencia de convergencia en 10. Otras alteraciones encontradas en menor proporción fueron estrabismo, ambliopía, conjuntivitis alérgica y daltonismo. Conclusiones: En los niños con dificultades escolares consideramos importante realizar una exploración oftalmológica exhaustiva que incluya las vergencias y la acomodación con el fin de identificar factores visuales que pudieran interferir en el proceso de aprendizaje.
\end{abstract}

Palabras clave: Errores refractivos. Insuficiencia acomodativa. Dificultades de aprendizaje. Niños.

\section{Abstract}

Background: The first cause of school failure is learning disabilities. Diagnosis and treatment require a multidisciplinary approach in which it is important to rule out any ophthalmological condition that can interfere with the learning process. There is no data about it in Mexican population; therefore, it is important to evaluate the most common visual defects. Purpose: To describe ophthalmological findings in children with learning difficulties. Method: The following data was retrieved from a retrospective review of the medical records of children aged 5 to 16 years with learning difficulties who underwent through ophthalmic examination

Correspondencia:

*Andrea Rangel-Padilla

Batallón de San Patricio 112, 1ํo. ote.

Col. Real de San Agustín, 66278

Fecha de recepción: 12-04-2021

Fecha de aceptación: 02-09-2021

DOI: 10.24875/RMO.M21000209
Disponible en internet: 03-01-2022

Rev Mex Oftalmol. 2022;96(1):3-8

www.rmo.com.mx

E-mail: andrearp@tec.mx

ublicado por Permanyer. Este es un artículo open access bajo la licencia CC BY-NC-ND (h187-4519/@ 2021 Sociedad Mexicana de Oftalm 
from January 2018 through January 2019. The records include the following variables: age, gender, visual acuity, ocular alignment, fusion, stereoacuity, near point of convergence, color vision, accommodative amplitude, cycloplegic refraction, slit lamp, fundus exam, and diagnosis. Results: Data of 69 children were included, their mean age was 9.3 years $( \pm 3.5), 51$ were men. Of the 69 children, 38 had an ophthalmic finding: 22 patients presented significant refractive error, 10 patients had accommodative insufficiency, 10 patients showed convergence insufficiency. Other diagnoses found were strabismus, amblyopia, allergic conjunctivitis, and color blindness. Conclusions: It is advisable that every child with learning difficulties should go for an exhaustive eye exam including accommodation and vergence tests to rule out any visual defects that can interfere with learning.

Keywords: Refractive errors. Accommodative Insufficiency. Learning disabilities. Children.

\section{Introducción}

Los problemas de aprendizaje se consideran la primera causa de fracaso escolar ${ }^{1}$. Estos constituyen un grupo heterogéneo de trastornos por los cuales niños con una inteligencia promedio tienen problemas en el procesamiento de la información o en la formulación de respuestas, afectando los procesos neurocognitivos, lo cual se manifiesta como déficit en pronunciación, comprensión, lecto-escritura, resolución de problemas matemáticos y organización de la información².

La visión desempeña un papel fundamental en la adquisición de conocimiento y en el desarrollo de habilidades como el lenguaje, la comunicación y la conexión con el entorno social y espacial, así como de aptitudes que requieren coordinación ojo-mano ${ }^{3-5}$.

A pesar de que la visión es fundamental para las actividades académicas, hoy en día se reconoce que el aprendizaje es un proceso cerebral complejo y, por lo tanto, un déficit visual específico no se relaciona de forma directa como la causa de un trastorno en el aprendizaje ${ }^{2,6-8}$.

Se ha descrito que los problemas oculares y académicos pueden coexistir ${ }^{2}$. Algunos niños con y sin dificultades en el aprendizaje suelen evitar actividades que impliquen utilizar la visión cercana por presentar síntomas como visión borrosa, diplopía, cefalea, sensación de tirantez de los ojos, pérdida de concentración y somnolencia ${ }^{1,9-11}$.

Algunos trastornos de la visión binocular, como son la insuficiencia acomodativa y la insuficiencia de convergencia, así como los errores de refracción no corregidos, pueden entorpecer la lectura e influir en el rendimiento académico ${ }^{2,11-16}$. Aunque la acomodación tiene un papel muy importante en la visión, no se evalúa sistemáticamente en los niños porque se supone que esta función es excelente en ellos; su valor promedio en los menores de 10 años es de alrededor de 14 dioptrías ${ }^{2,14}$.

Las dificultades en el aprendizaje requieren un abordaje multidisciplinario para su diagnóstico y tratamiento, en el que se involucran educadores, neuropsicólogos, médicos de diferentes especialidades y la familia. Dentro de la batería de estudios se encuentra la valoración oftalmológica con el objetivo de descartar algún problema asociado que pueda interferir en el proceso de aprendizaje ${ }^{17}$.

Existe poca información sobre el estado visual de los pacientes con dificultades del aprendizaje sin trastornos específicos, como dislexia, déficit de atención e hiperactividad, entre otros ${ }^{16}$.

El objetivo principal de este estudio es describir las alteraciones oftalmológicas encontradas en un grupo de pacientes mexicanos con dificultades de aprendizaje remitidos a la consulta para su valoración oftalmológica.

\section{Método}

Se realizó un estudio observacional y transversal con población mestiza de la ciudad de Monterrey, Nuevo León, en el cual se incluyeron 69 pacientes sin alteraciones neurológicas clínicamente evidentes, con edades comprendidas entre 5 y 16 años, remitidos de un centro de apoyo educativo para valoración oftalmológica por presentar dificultades de aprendizaje, durante el periodo de enero de 2018 a enero de 2019.

Las variables estudiadas fueron la presencia o ausencia de alguna alteración oftalmológica, como errores de refracción, insuficiencia acomodativa, insuficiencia de convergencia, ambliopía, estrabismo, alteración en la visión de colores y otras patologías oftalmológicas. Para ello, se recopilaron de los expedientes electrónicos los siguientes datos: sexo, edad, agudeza visual, refracción ciclopléjica, motilidad ocular, sensorialidad, estereoagudeza, punto próximo de convergencia, amplitudes acomodativas, presión intraocular, biomicroscopía, fundoscopía y diagnóstico. Toda la información se registró en una base de datos para posteriormente ser analizada mediante estadística descriptiva para los datos clínicos y demográficos, frecuencias y porcentajes para las variables cualitativas, 
y medias y medianas (desviación estándar y rangos) para las variables cuantitativas de acuerdo con su distribución, utilizando el programa SPSS Statistics for Windows, Versión 25.0, IBM.

El estudio fue aprobado por el Comité de Ética en Investigación de la Escuela de Medicina del Instituto Tecnológico y de Estudios Superiores.

A todos los pacientes que acudieron a valoración se les realizaron los siguientes procedimientos: toma de agudeza visual mediante optotipos estandarizados de acuerdo con la edad a 6 metros de distancia; valoración de la motilidad ocular mediante prueba de pantalleo o cover test a 6 metros y $40 \mathrm{~cm}$ en posición primaria para detectar la presencia de forias y tropías, y se realizaron versiones y ducciones mediante seguimiento visual con un objeto de fijación. Además, se midió el punto próximo de convergencia y se evaluaron la sensorialidad con la prueba de Worth 4 dot y la estereoagudeza mediante Random dot test. Se evaluó la visión de colores con láminas de Isihara y se midieron las amplitudes acomodativas con el método de Sheard. La toma de presión intraocular se realizó con un tonómetro de rebote. Se realizó una valoración del segmento anterior con lámpara de hendidura para detectar patología de la superficie ocular; posterior a la aplicación en cada ojo de dos gotas de solución oftálmica de ciclopentolato al $1 \%$ y tropicamida $8 \mathrm{mg} / \mathrm{ml}$ con fenilefrina $50 \mathrm{mg} / \mathrm{ml}$ se realizó refracción ciclopléjica. Finalmente, se llevó a cabo la evaluación del fondo de ojo mediante oftalmoscopía indirecta.

Se determinó la presencia de error de refracción significativo que requiere prescripción en caso de encontrar miopía $\geq-0.75 \mathrm{D}$, hipermetropía $\geq+3.50 \mathrm{D}$, astigmatismo $\geq-1.00 \mathrm{D}$ y anisometropía $\geq 1.50 \mathrm{D}$ en cualquier tipo de error refractivo6,18-20; insuficiencia acomodativa cuando el valor encontrado fue dos dioptrías por debajo del valor esperado de acomodación para la edad utilizando la fórmula de Hofstetter, $15-(0.25 \times \text { edad del paciente })^{11,21}$; insuficiencia de convergencia ante la presencia de exodesviación mayor de 4 DP más la presencia de un punto próximo de convergencia alejado $\geq 7 \mathrm{~cm}^{11}$; ambliopía de acuerdo con los criterios establecidos por la American Academy of Ophthalmology22; estrabismo ante la presencia de tropía en la prueba de pantalleo o cover uncover test; alteración en la visión de colores ante el resultado anormal del test de Isihara; y otras patologías oftalmológicas en caso de encontrar alteraciones en la biomicroscopía o la fundoscopía.
Tabla 1. Características demográficas de la población estudiada

\begin{tabular}{|l|c|}
\hline Característica & $N=69$ \\
\hline Edad promedio (DE), años & $9.3 \pm 3.5$ \\
\hline Sexo, n (\%) & \\
Masculino & 51 \\
Femenino & 18 \\
\hline
\end{tabular}

DE: desviación estándar.

Tabla 2. Diagnósticos oftalmológicos encontrados

\begin{tabular}{|l|c|}
\hline Diagnóstico & $N=69$ \\
\hline Sano & 31 \\
\hline Error refractivo significativo & 22 \\
\hline Insuficiencia acomodativa & 10 \\
\hline Insuficiencia de convergencia & 10 \\
\hline Estrabismo & 4 \\
\hline Ambliopía & 4 \\
\hline Daltonismo & 4 \\
\hline Otros & 10 \\
\hline
\end{tabular}

En 18 niños se encontró más de un diagnóstico oftalmológico.

\section{Resultados}

Se incluyeron los datos de 69 pacientes que fueron remitidos de una institución educativa tras una evaluación psicopedagógica por presentar dificultades de aprendizaje para valoración oftalmológica a la unidad de oftalmología pediátrica y estrabismo del Hospital Zambrano Hellion, en el periodo del 1 enero de 2018 al 1 de enero de 2019.

La edad promedio de los pacientes fue de $9.3 \pm 3.5$ años (rango: 5-16 años) y 51 eran de sexo masculino (Tabla 1). En 31 de los 69 pacientes no se encontraron alteraciones oftalmológicas, 22 presentaron error de refracción que requirió corrección óptica, en 10 se encontró insuficiencia acomodativa y en 10 insuficiencia de convergencia, 4 presentaron estrabismo y 4 ambliopía (en 3 de ellos de causa estrábica y en 1 anisometrópica), 4 se diagnosticaron de daltonismo y 10 tuvieron diferentes diagnósticos, como conjuntivitis alérgica y blefaroconjuntivitis estafilocócica, entre otros (Tabla 2 y Fig. 1).

La agudeza visual LogMAR promedio en el ojo derecho fue de $0.13( \pm 0.17)$ y en el ojo izquierdo de 0.12 ( \pm 0.17). De los 22 pacientes con error de 


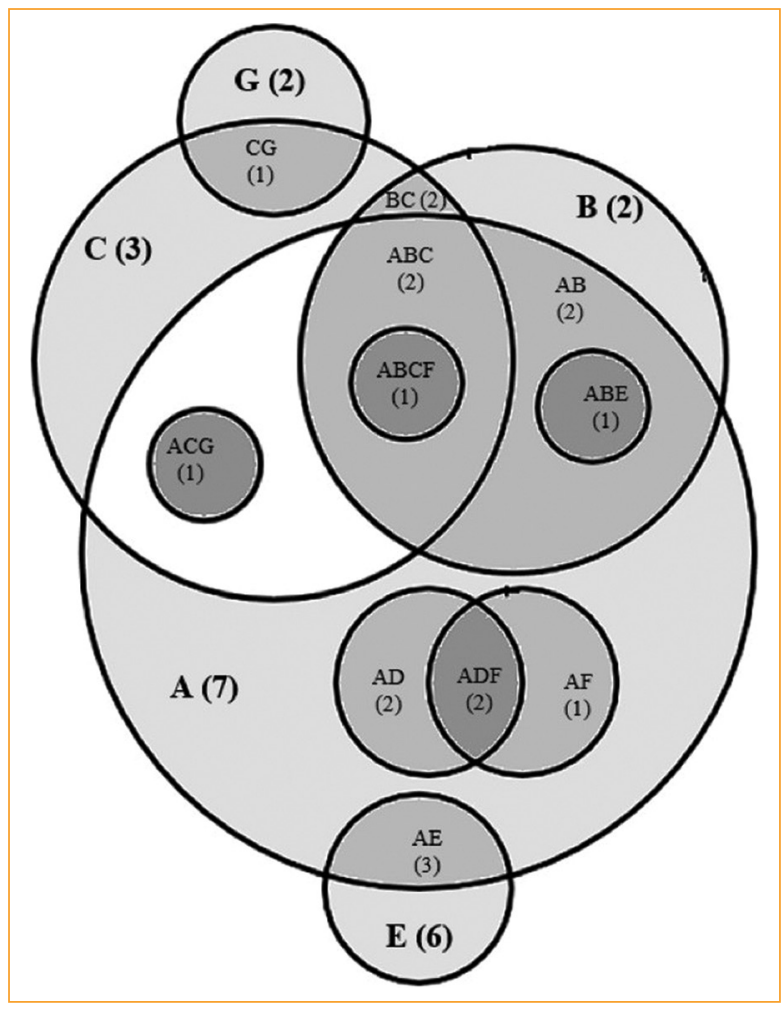

Figura 1. A: error refractivo significativo. B: insuficiencia acomodativa. C: insuficiencia de convergencia.

D: ambliopía. E: otros. F: estrabismo. G: daltonismo. Entre paréntesis el número de casos.

refracción significativo que ameritó corrección óptica, 6 tuvieron hipermetropía, 11 astigmatismo hipermetrópico, 3 miopía y 2 astigmatismo miópico (Tabla 3).

De los 10 pacientes con insuficiencia acomodativa, 6 tuvieron un error refractivo significativo que ameritó corrección óptica, 5 presentaron insuficiencia de convergencia añadida y 1 estrabismo. La media de amplitud de acomodación de los pacientes catalogados con insuficiencia acomodativa fue de -7.07 dioptrías $( \pm 1.45)$ en el ojo derecho y de -7.75 dioptrías $( \pm 1.16)$ en el ojo izquierdo, presentando una diferencia media de 4.88 dioptrías (4 a 6.5) de acuerdo con lo que les correspondería por la edad según la fórmula de Hofstetter. La media de acomodación del total de los niños estudiados fue de 10.14 D ( \pm 1.78$)$ en el ojo derecho y de 10.23 $\mathrm{D}( \pm 1.58)$ en el ojo izquierdo (Tabla 4 y Fig. 2).

\section{Discusión}

Los resultados expuestos corresponden a una pequeña muestra de población mestiza del noreste de México, y más de la mitad de los pacientes presentaron
Tabla 3. Errores de refracción

\begin{tabular}{|l|c|}
\hline Error de refracción & N $=22$ \\
\hline Hipermetropía & 6 \\
\hline Astigmatismo hipermetrópico & 11 \\
\hline Miopía & 3 \\
\hline Astigmatismo miópico & 2 \\
\hline
\end{tabular}

Tabla 4. Amplitudes acomodativas encontradas en niños con insuficiencia acomodativa

\begin{tabular}{|l|l|}
\hline Insuficiencia acomodativa $(\mathbb{N}=10)$ & Media (DE) \\
\hline Amplitud acomodativa ojo derecho & $7.07( \pm 1.45)$ \\
\hline Amplitud acomodativa ojo izquierdo & $7.75( \pm 1.16)$ \\
\hline Diferencia esperada de acuerdo con la edad & $4.88(4-6.5)$ \\
\hline
\end{tabular}

DE: desviación estándar.

alteraciones oftalmológicas que podrían dificultar las actividades académicas ${ }^{2,8,16}$.

La edad promedio de los pacientes fue de $9.3 \pm 3.5$ años (rango: 5-16 años). Las recomendaciones actuales sobre revisiones oftalmológicas en los niños mencionan la importancia de realizarlas de forma temprana y periódica ${ }^{14}$. La mayoría de los pacientes fueron del sexo masculino; sin embargo, otro estudio realizado en pacientes con dificultades de aprendizaje encontró en sus resultados que no existen diferencias significativas cuando se consideran trastornos de aprendizaje en general ${ }^{23}$.

Se ha descrito que un $5-25 \%$ de los pacientes en edad escolar presentan algún problema oftalmológico $^{2,18}$. Las principales alteraciones oftalmológicas encontradas fueron los errores de refracción en 22 de los 69 participantes, lo que corresponde a una tercera parte de la muestra. La prevalencia varía en función de la edad y de la población estudiada; por ejemplo, en la India, un metaanálisis reveló que la prevalencia global de errores de refracción en niños era del $8 \%$, específicamente miopía $\geq 2$ D en el $5.3 \%$ de la población, hipermetropía $\geq 2 \mathrm{D}$ en el $4 \%$ de la población y astigmatismo $\geq 2 \mathrm{D}$ en el $5.4 \%$ de la población ${ }^{24}$. La American Academy of Ophthalmology, en su guía de práctica clínica sobre evaluaciones oftalmológicas en niños publicada en el año 2017, reportó una prevalencia de miopía $\geq-0.75 \mathrm{D}$ en niños de $5-17$ años del $0.7 \%$ al $9 \%$, de hipermetropía $\geq+3.00 \mathrm{D}$ del $4 \%$ al $9 \%$, y de 


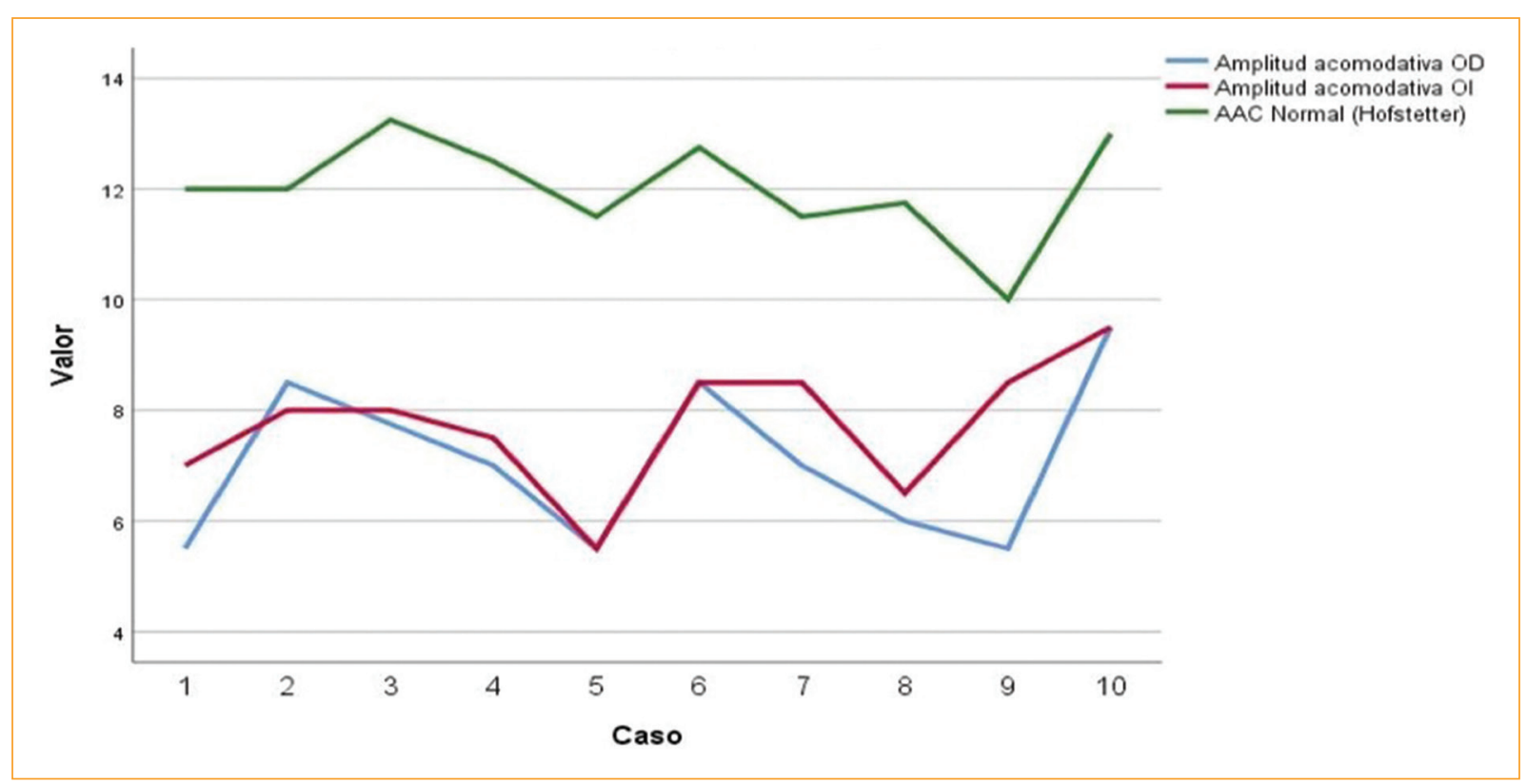

Figura 2. Valor medido de amplitudes acomodativas en niños con insuficiencia acomodativa en dioptrías y el esperado en cada caso según la edad.

astigmatismo $\geq 1.5 \mathrm{D}$ del $4 \%$ al $11 \%$ en la población pediátrica ${ }^{18}$. En cuanto a la frecuencia de estrabismo y de ambliopía, también se encuentra una proporción ligeramente superior a la reportada en la literatura científica ${ }^{18}$.

Es reconocido que las alteraciones acomodativas y de convergencia son infrecuentes en los niños ${ }^{2,16}$. Un estudio realizado con 292 niños portugueses de 10 a 14 años de edad encontró una prevalencia de insuficiencia de convergencia del $6.8 \%$ y de insuficiencia acomodativa del $10 \%$, y la coexistencia de ambas en un $3 \%{ }^{21}$. Otro estudio realizado en Sudáfrica reportó una prevalencia del $4.3 \%$ de insuficiencia de convergencia ${ }^{25}$, y otro realizado en Ecuador halló una prevalencia de insuficiencia acomodativa del $16 \%{ }^{26}$. En este estudio encontramos 10 pacientes de 69 (14.5\%) con insuficiencia acomodativa y $10(14.5 \%)$ con insuficiencia de convergencia, y en la mitad de los casos la coexistencia de ambas, de modo similar a lo reportado por Davis, et al. ${ }^{11}$, quienes encontraron en su muestra poblacional una prevalencia mayor tanto de insuficiencia acomodativa como de convergencia, y la coexistencia de ambas en la mitad del total de los casos. Consideramos necesarias futuras investigaciones en el área para determinar si esto constituye un factor de riesgo o no para presentar dificultades en el aprendizaje, pues ambas se han descrito como causantes de síntomas astenópicos en la población infantil ${ }^{16,21}$.

\section{Conclusiones}

Este estudio encontró un número importante de alteraciones oftalmológicas, por lo que consideramos importante fomentar los programas de tamizaje visual periódico desde la infancia, así como una exploración oftalmológica exhaustiva que incluya las vergencias y la acomodación en niños con dificultades escolares, con el fin de identificar alteraciones visuales que pudieran interferir en el proceso de aprendizaje.

Los errores de refracción, la insuficiencia acomodativa y la insuficiencia de convergencia fueron las alteraciones oftalmológicas encontradas con mayor frecuencia en esta población. La coexistencia de insuficiencia acomodativa e insuficiencia de convergencia fue representativa, y ambas requieren mayor investigación.

\section{Financiamiento}

Los autores declaran que para esta investigación no se han utilizado fuentes externas de financiamiento para la realización de este estudio.

\section{Conflicto de intereses}

Los autores declaran no tener conflicto de intereses. 


\section{Responsabilidades éticas}

Protección de personas y animales. Los autores declaran que para esta investigación no se han realizado experimentos en seres humanos ni en animales.

Confidencialidad de los datos. Los autores declaran que han seguido los protocolos de su centro de trabajo sobre la publicación de datos de pacientes.

Derecho a la privacidad y consentimiento informado. Debido a la naturaleza del estudio, observacional retrospectivo, no se utilizó consentimiento informado, los datos personales de los sujetos mencionados fueron protegidos en todo momento.

\section{Bibliografía}

1. Dusek WA, Pierscionek BK, McClelland JF. An evaluation of clinical treatment of convergence insufficiency for children with reading difficulties. BMC Ophthalmol. 2011;11:21.

2. Handler SM, Fierson WM. Reading difficulties and the pediatric ophthalmologist. J AAPOS. 2017;21:436-42.

4. Van Leeuwen LM, Rainey L, Kef S, Van Rens GHMB, Van Nispen RMA Investigating rehabilitation needs of visually impaired young adults according to the International Classification of Functioning, Disability and Health. Acta Ophthalmol. 2015;93:642-50.

5. Day S. Normal and abnormal visual development. En: Pediatric ophthalmology. Oxford UK: Blackwell Science; 1997. p. 13-28.

6. Healy A, Erenberg G, Kaminer RK, La Camera R, Nackashi JA, Poncher JR, et al. Learning disabilities, dyslexia, and vision. Pediatrics. 1992;90:124-6

7. Flax N. Visual function in learning disabilities. J Learn Disabil. 1968;1:551-6.

8. Creavin AL, Lingam R, Steer $C$, Williams $C$. Ophthalmic abnormalities and reading impairment. Pediatrics. 2015;135:1057-65.

9. Borsting E, Mitchell GL, Kulp MT, Scheiman M, Amster DM, Cotter S, et al. Improvement in academic behaviors following successful. Optom Vis Sci. 2012;89:12-8.

10. Scheiman M, Cotter S, Mitchell GL, Kulp M, Rouse M, Hertle R, et al Randomized clinical trial of treatments for symptomatic convergence insufficiency in children. Arch Ophthalmol. 2008;126:1336-49.
11. Davis AL, Harvey EM, Twelker JD, Miller JM, Leonard-Green T, Campus I. Convergence insufficiency, accommodative insufficiency, visual symptoms, and astigmatism in Tohono O'odham students. J Ophthalmol. 2016;2016:6963976.

12. Scheiman M, Rouse M, Kulp MT, Cotter S, Hertle R, Lynn Mitchell G, et al. Treatment of convergence insufficiency in childhood: a current perspective NIH Public Access Author Manuscript. Optom Vis Sci. 2009;86:420-8.

13. Bruce AS, Lakkis G. Accommodation deficiency in healthy young individuals. J Pediatr Ophthalmol Strabismus. 1990;27:327.

14. Prangen AD. Subnormal accommodation. Trans Am Ophthalmol Soc. 1931;29:372-91.

15. Cacho-Martínez P, Cantó-Cerdán M, Carbonell-Bonete S, García-Muñoz A. Characterization of visual symptomatology associated with refractive, accommodative, and binocular anomalies. J Ophthalmol. 2015:2015:31-42.

16. Phillips $\mathrm{PH}$. Pediatric ophthalmology and childhood reading difficulties: convergence insufficiency: relationship to reading and academic performance. J AAPOS. 2017;21:444-6.e1.

17. Slavin RE, Collins ME, Repka MX, Friedman DS, Mudie LI, Owoeye JO, et al. In plain sight: reading outcomes of providing eyeglasses to disadvantaged children. J Educ Students Placed Risk. 2018;23:250-8.

18. Wallace DK, Morse CL, Melia M, Sprunger DT, Repka MX, Lee KA, et al. Pediatric Eye Evaluations Preferred Practice Pattern®: I. Vision screening in the primary care and community setting; II. Comprehensive ophthalmic examination. Ophthalmology. 2018;125:184-227.

19. Macewen C. The Royal College of Ophthalmologists Seminar Programme organised and Chaired by The Royal College of Physicians. 2013;(March):4-5.

20. Leat SJ. To prescribe or not to prescribe? Guidelines for spectacle prescribing in infants and children. Clin Exp Optom. 2011;94:514-27.

21. Nunes AF, Monteiro PML, Ferreira FBP, Nunes AS. Convergence insufficiency and accommodative insufficiency in children. BMC Ophthalmol. 2019;19:1-8.

22. Wallace DK, Repka MX, Lee KA, Melia M, Christiansen SP, Morse CL, et al. Amblyopia Preferred Practice Pattern®. Ophthalmology. 2018;125:105-42.

23. Moll K, Kunze S, Neuhoff N, Bruder J, Schulte-Körne G. Specific learning disorder: prevalence and gender differences. PLoS One. 2014;9:e103537.

24. Sheeladevi S, Seelam B, Nukella PB, Modi A, Ali R, Keay L. Prevalence of refractive errors in children in India: a systematic review. Clin Exp Optom. 2018;101:495-503.

25. Wajuihian SO, Hansraj R. Anomalías de vergencia en una muestra de estudiantes de secundaria de Sudáfrica. J Optom. 2016;9:246-57.

26. Legrá Nápoles S, Galarza Núñez JL, Martínez Herrera CP Gallo González M. Disfunciones acomodativas en estudiantes no estrábicos de la Unidad Educativa Arturo Borja, Orellana, Ecuador. Rev Conrado. 2019;15:110-24. 